\section{A Simple Method for Designing High-Quality Prototype Filters for $M$-Band Pseudo QMF Banks}

\author{
Charles D. Creusere and Sanjit K. Mitra
}

\begin{abstract}
This correspondence discusses a new method for designing the prototype filters necessary to implement $M$-band pseudo QMF banks. This method does not rely on the traditional nonlinear optimization used in past work but rather optimizes a single parameter on a convex error surface, consistently delivering the best equiripple filter possible while minimizing the overlapped passband distortion.
\end{abstract}

\section{INTRODUCTION}

Shortly after Johnston introduced his two-band, linear phase quadrature mirror filter (QMF) bank in [1], Rothweiler [2] and Chu [3] independently developed $M$-band extensions to it. These have been called generalized or pseudo QMF banks and have also been extended to the nonuniform case in [4].

An $M$-band maximally decimated analysis filter bank consists of $M$ parallel bandpass filters followed by $M$-fold downsamplers, while the corresponding synthesis bank has an $M$-fold upsampler in each channel, followed by a bandpass filter and, finally, a summation of all the channels. There are three sources of distortion in the reconstructed output of such a filter bank--amplitude distortion, phase distortion, and aliasing. All of the filter banks given in [2]-[4] rely on the flatness and linear phase of their prototype filters as well as the orthogonality of their modulation functions (phase shifted sine waves) to ensure good amplitude and phase reconstruction. To reduce the distortion caused by aliasing, these filter banks cancel it between adjacent bands and assume that there is no aliasing between nonadjacent bands-i.e., that the stopband attenuation of a given analysis filter in all nonadjacent bands is infinite. While this is obviously not true for real filters, pseudo QMF banks work quite well in practice if the attenuation is sufficiently high compared to the output resolution required in the reconstructed signal.

For speech, Chu found that about $40 \mathrm{~dB}$ of attenuation in nonadjacent bands was sufficient to ensure an output signal of adequate quality [3]. For wideband audio, where input samples typically have 16-bit precision, far greater stopband attenuation is needed for highquality reconstruction. For example, in the digital compact cassette (DCC) music format, Phillips uses a 32-band pseudo QMF bank for signal analysis and coding with a stopband attenuation in nonadjacent bands of greater than $100 \mathrm{~dB}$, requiring an FIR prototype filter with 512 taps [5]. Unfortunately, the classical procedure for designing such prototype filters, as first developed in [1] for the two-band case and subsequently modified in [2]-[4] for the $M$-band case, requires optimization of a nonlinear cost function with many local minima. The larger the number of filter taps, the more difficult it is to find an initial starting point that allows the optimization to converge to a good filter. In [1], Johnston points out that even for the two-band filter bank, the optimization process requires significant human intervention

Manuscript received April 5, 1993; revised September 19, 1994. This work was supported by a fellowship from the U.S. Navy and by a University of California MICRO grant with matching support from Digital Instruments, Rockwell International, and Tektronics. The associate editor coordinating the review of this paper and approving it for publication was Prof. Sergio D. Cabrera.

C. D. Creusere is with Naval Air Warfare Center, China Lake, CA USA

S. K. Mitra is with the Uniersity of California at Santa Barbara, Santa Barbara, CA 93106 USA

IEEE Log Number 9409762. to obtain acceptable filters. Recently, Chen and Lee have devised an optimization technique based on linearization of the cost function, providing for more certain convergence and permitting the design of equiripple filters that satisfy the flatness constraint required for good amplitude reconstruction [6]. Unfortunately, this technique is both complicated and is only applicable to two-band filter banks. In another recent paper, the authors have developed a method for designing the prototype filters that avoids the optimization and instead relies on spectral factorization to generate the prototype filter [7] While this method eliminates the problematic nonlinear optimization, it is conceptually more difficult to understand and use than the method proposed here, and the filter banks it creates are not flat at $\omega=0$ and $\omega=\pi$. In this correspondence, we outline a new, very simple technique for generating $M$ th-band lowpass prototype filters for use in pseudo QMF banks, especially those requiring highquality signal reconstruction. This technique is presented in the next section, followed by a design example in Section III and conclusions in Section IV.

\section{Filter Design Technique}

To get high-quality reconstruction in a pseudo QMF bank, the lowpass prototype $H(\omega)$ must satisfy as much as possible two conditions:

$$
|H(\omega)|^{2}+\left|H\left(\omega-\frac{\pi}{M}\right)\right|^{2}=1, \quad \text { for } 0<\omega<\frac{\pi}{M}
$$

and

$$
|H|(\omega) \mid=0, \quad \text { for } \omega>\frac{\pi}{M} .
$$

If (1) is satisfied exactly, all amplitude distortion is eliminated in the combined analysis/synthesis system, while if (2) is satisfied, there is no aliasing between nonadjacent bands. Aliasing between adjacent bands, on the other hand, is eliminated by selecting appropriate phase factors in the modulation [2]. The modulated analysis and synthesis filters are then given by

$$
\begin{gathered}
h_{i}(n)=\left\{\begin{array}{c}
\operatorname{Re}\left(a_{i}\right) \cos [\pi(2 i+1)(2 n+1) / 4 M] \\
-\operatorname{Im}\left(a_{i}\right) \sin [\pi(2 i+1)(2 n+1) / 4 M]
\end{array}\right\} h(n) \\
0 \leq i \leq M
\end{gathered}
$$

and

$$
\begin{gathered}
g_{i}(n)=\left\{\begin{array}{c}
\operatorname{Re}\left(a_{i}\right) \cos [\pi(2 i+1)(2 n+1) / 4 M] \\
+\operatorname{Im}\left(a_{i}\right) \sin [\pi(2 i+1)(2 n+1) / 4 M]
\end{array}\right\} h(n) \\
0 \leq i \leq M
\end{gathered}
$$

respectively, where the phase factor $a_{i}$ is

$$
a_{i}=\frac{\sqrt{2}}{2} \cdot \begin{cases}1+j, & i \text { even } \\ 1-j, & i \text { odd. }\end{cases}
$$

Unfortunately, it is not possible to design a finite length filter that exactly satisfies the constraints of (1) and (2), so it is necessary instead to design one that approximately satisfies them. The methods of [1]-[4] try to do this directly by combining (1) and (2) into a single cost function that is then minimized using the Hooke and Jeaves algorithm. Our method uses the Parks-McClellan algorithm [8] to directly design filters that approximately satisfy (1) and (2) by varying the passband edge. The filter length, relative error weighting, and stopband edge are fixed before the optimization procedure is started, while the passband edge is adjusted to minimize

$$
\begin{aligned}
& \phi=\max _{\omega}\left\{|H(\omega)|^{2}+\left|H\left(\omega-\frac{\pi}{M}\right)\right|^{2}-1\right\}, \\
& 0<\omega<\frac{\pi}{M} .
\end{aligned}
$$




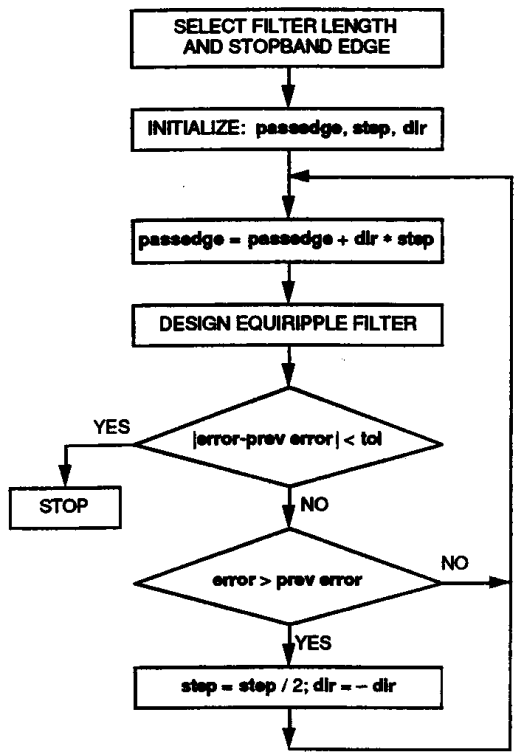

Fig. 1. Flowchart of the proposed optimization algorithm.

We have found experimentally that this cost function is convex with respect to the passband edge. Thus, any reasonable optimization routine will converge to the same global minima regardless of the initial starting value of the passband edge. A flowchart of the complete optimization routine is shown in Fig. 1. This algorithm simply adjusts the passband edge at each iteration by the stepsize denoted by step, designs a new equiripple filter (using the Parks-McClellan algorithm), and computes the error, $\phi$, (denoted error) using (6) as the cost function. Whenever the error goes up over that of the previous iteration (called prev error), step is halved and the search direction labeled dir is changed. The optimization process is halted when the difference between the error of the current iteration and that of the previous iteration is within a specified tolerance (called tol). While we could have used a gradient descent algorithm instead of this one, it would have been very slow because approximation of the gradient (via a finite difference) would have required two evaluations of the cost function at each step. Since cost function evaluations entail the design of complete equiripple filters, they are very expensive and should be done as seldom as possible. Using the proposed algorithm, we avoid this problem somewhat since we only have to perform extra cost function evaluations when the search direction is changed.

Because this algorithm only minimizes the overlapped passband ripple given by (6) and since the stopband edge is fixed at $\pi / M$, the length of the filter must be chosen such that the filter has enough stopband attenuation to approximate (2) effectively. We have found that the best way to do this is to fix the passband edge at $\pi / 2 M$ (with the stopband fixed at $\pi / M$ ) and select the filter length such that the desired attenuation in the stopband is achieved. In the course of the optimization procedure, the passband edge will generally move toward zero, slightly increasing the stopband attenuation. We have also found it useful in this optimization to weight the passband error more heavily than the stopband error when invoking the Parks-McClellan algorithm. In one example, we found that a weighting of ten to one halved the final filter's cost ( $\phi_{\mathrm{opt}}$ as expressed by (6)) while only reducing the stopband attenuation by less than $1 \mathrm{~dB}$. We have considered adding an outer loop to the procedure shown in Fig. 1 to optimize the filter coefficients over both the weighting factor and $\phi$ (fixing first the weighting factor,

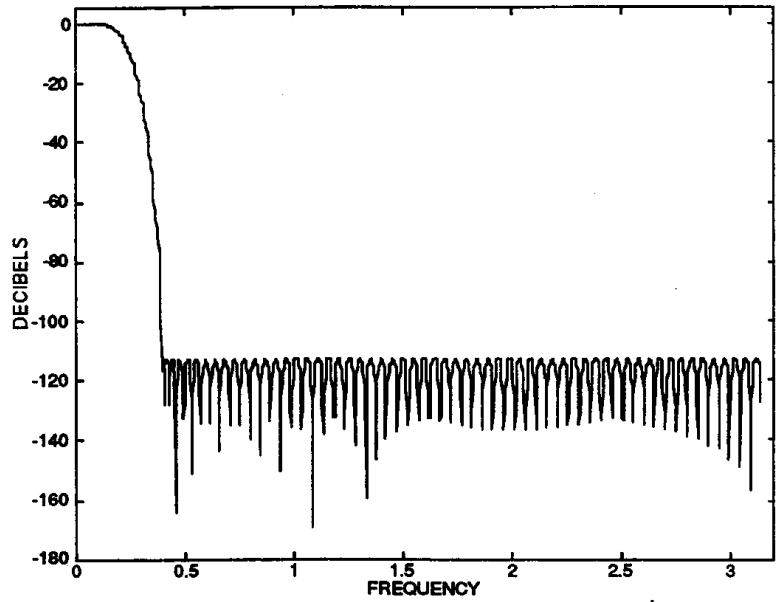

Fig. 2. Frequency response of prototype filter for an eight-band pseudo QMF bank. This filter was designed with the passband error being weighted five times more than the stopband error.

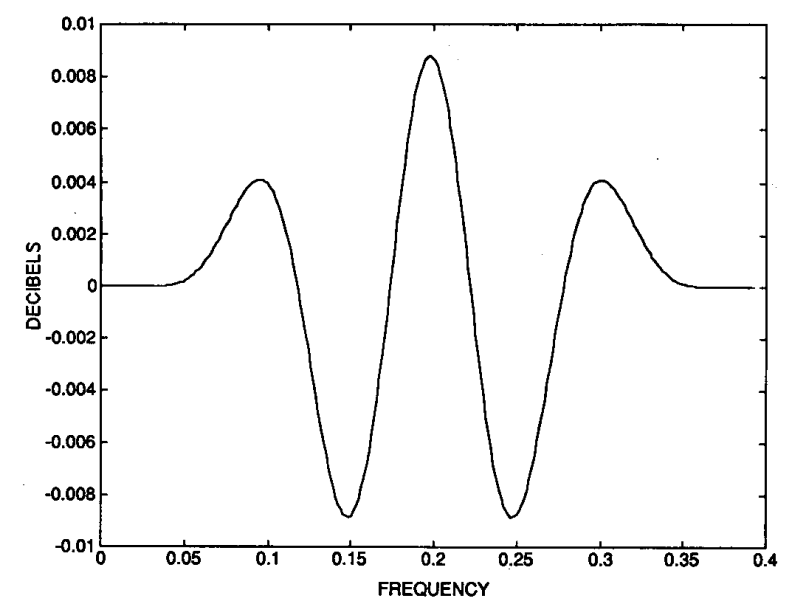

Fig. 3. Passband ripple of the eighth-band filter overlapped with itself shifted by $\pi / 8\left(\phi_{\mathrm{opt}}+1\right)$.

optimizing $\phi$ and then vice versa with the process repeating until convergence is achieved). Unfortunately, while this procedure does improve the quality of the final prototype filter slightly, it also greatly increases the convergence time of the algorithm. Consequently, for our design examples we have chosen to fix the weighting and optimize only over $\phi$ using the procedure outlined in Fig. 1.

\section{DESIGN EXAMPLES}

We demonstrate now the operation of the previously described method by using it to design two different lowpass prototype filters: an eighth-band filter and a 32nd-band filter. Both filters were designed for high quality signal reconstruction with a stopband attenuation of greater than $100 \mathrm{~dB}$, leading us to select filter lengths of 128 for the eighth-band filter and $\mathbf{5 1 2}$ for the 32nd-band filter. The magnitude response of the eighth-band filter is shown in Fig. 2 and its overlapped passband response, $\phi_{\mathrm{opt}}+1$, is shown in Fig. 3. The stopband attenuation of the eighth-band filter is about $116 \mathrm{~dB}$ while that of the 32nd-band filter is $118 \mathrm{~dB}$; the maximum deviation from unity in their overlapped passbands are 0.008 and $0.007 \mathrm{~dB}$, respectively. Note 


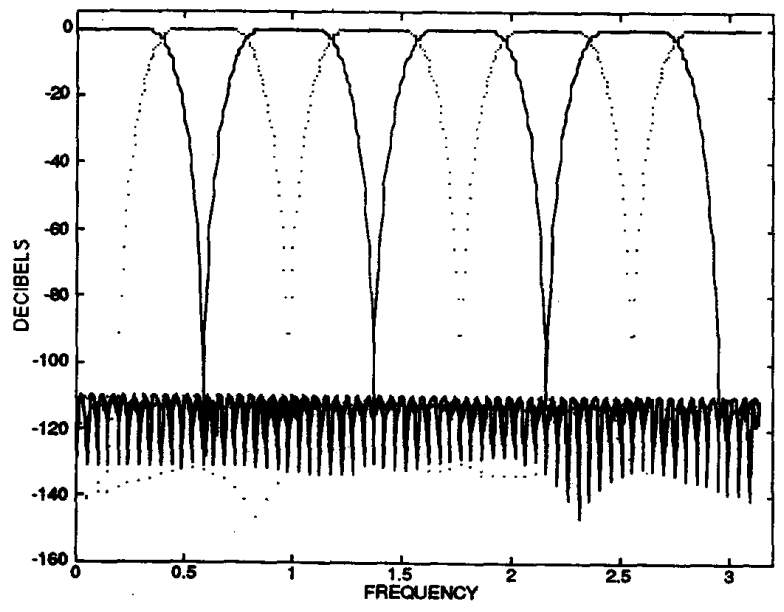

Fig. 4. Frequency responses of all eight filters in an eight-band pseudo QMF bank. Alternating solid and dotted lines have been used for clarity.

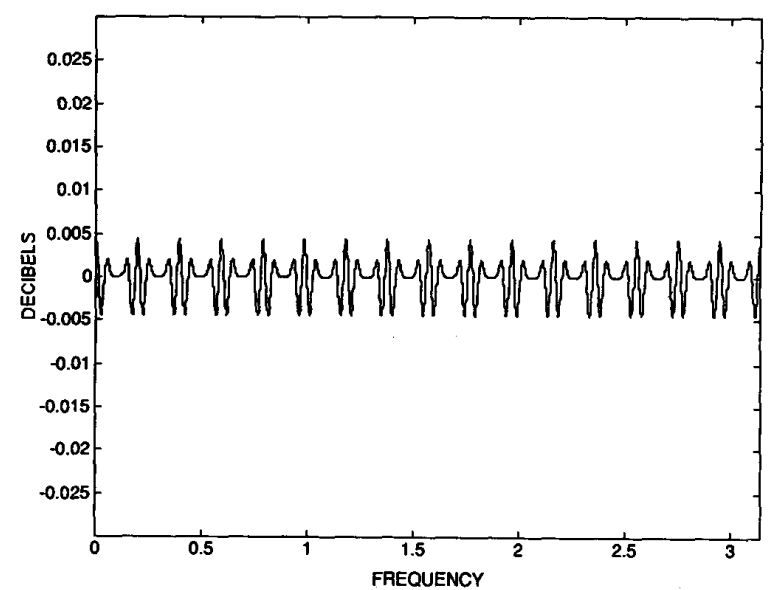

Fig. 5. Overall frequency response of eight-band analysis/synthesis system.

from Fig. 3 that the error in the overlapped passband is equiripple (which is also the case for all other filters designed with this method), indicting that we have, indeed, minimized the cost function of (6). For the actual eight-band analysis bank shown in Fig. 4, we see that the transition bands of adjacent filters overlap but that those of nonadjacent filters do not. In addition, the frequency response for the complete analysis/synthesis system is very flat (see Fig. 5) with no large dips or bulges at $\omega=0$ or $\omega=\pi$.

We have also designed an eighth-band lowpass filter with 48 taps and compared it to a similar filter designed using the conventional nonlinear optimization process in [4]. Our filter has a higher stopband attenuation than theirs, but its overlapped passband error is 0.11 $\mathrm{dB}$ compared with $0.016 \mathrm{~dB}$ for their filter. While it is difficult to say which of these filters would provide the best results in a real application, our method of filter design is far easier to implement and converges with much more certainty to a good result than does theirs. It is also shown in [4] how to use multiple stages of pseudo QMF banks to create nonuniform maximally decimated filter banks. Filters designed using the proposed method can be substituted for those in [4] and used to create nonuniform filter banks since they have the same salient properties.

\section{CONCLUSION}

We have described here a very simple algorithm for designing lowpass prototype filters suitable for use in pseudo QMF banks. To illustrate the applicability of this algorithm, we have designed two different filters, both for such applications as wideband audio coding that require high quality reconstructed signals. As pointed out in [4], such filters cannot be easily designed using the conventional nonlinear technique because they are too long, leading to cost functions that have too many local minima. By contrast, the quality of the solution found with our method does not go down as the filter length increases - in fact, it seems to get slightly better. We feel that this filter design method is very valuable because of its conceptual simplicity and because of the ease with which it can be implemented using the widely available Parks-McClellan algorithm.

\section{REFERENCES}

[1] J. D. Johnston, "A filter family designed for use in quadrature mirror filter banks," in Proc. IEEE Int. Conf. Acoust., Speech, Signal Processing, Denver, CO, 1980, pp. 291-294.

[2] J. H. Rothweiler, "Polyphase quadrature filters-A new subband coding technique," in Proc. IEEE Int. Conf. Acoust., Speech, Signal Processing, Boston, MA, 1983, pp. 1280-1283.

[3] P. L. Chu, "Quadrature mirror filter design for an arbitrary number of equal bandwidth channels," IEEE Trans. Acoust., Speech, Signal Processing, vol. ASSP-33, no. 1, pp. 203-218, Feb. 1985.

[4] R. V. Cox, "The design of uniformly and nonuniformly spaced pseudoquadrature mirror filters," IEEE Trans. Acoust., Speech, Signal Processing, vol. ASSP-34, no. 5, pp. 1090-1096, Oct. 1986.

[5] G. C. P. Lokhoff, "Precision adaptive coding for the digital compact cassette," IEEE Trans. Consumer Electron., vol. 38, no. 4, pp. 784-789, Nov. 1992.

[6] C. Chen and J. Lee, "Design of quadrature mirror filters with linear phase in the frequency domain-Part II," IEEE Trans. Circuits Syst /Video Technol]., vol. 39, no. 9, pp. 593-605, Sept. 1992.

[7] R. D. Koilpillai and P. P. Vaidyanathan, "A spectral factorization approach to pseudo-QMF design," IEEE Trans. Signal Processing, vol. 41, no. 1, pp. 82-92, Jan. 1993.

[8] A. V. Oppenheim and R. W. Schafer, Discrete-Time Signal Processing. Englewood Cliffs, NJ: Prentice-Hall, 1989, pp. 464-488. 